\title{
Characteristics of Long-Duration Inhibitory Postsynaptic Potentials in Rat Neocortical Neurons In Vitro
}

\author{
James R. Howe, ${ }^{1,2}$ Bernd Sutor, ${ }^{1}$ and Walter Zieglgänsberger ${ }^{1}$ \\ Received April 25, 1985; revised September 8, 1986; accepted September 1986 \\ KEY WORDS: inhibitory postsynaptic potential (IPSP); potassium conductance; neocortex; stimulation \\ fatigue; intracellular recordings.
}

\section{SUMMARY}

1. The characteristics of long-duration inhibitory postsynaptic potentials (1-IPSPs) which are evoked in rat frontal neocortical neurons by local electrical stimulation were investigated with intracellular recordings from an in vitro slice preparation.

2. Stimulation with suprathreshold intensities evoked l-IPSPs with typical durations of $600-900 \mathrm{msec}$ at resting membrane potential. Conductance increases of $15-60 \%$ were measured at the peak amplitude of 1-IPSPs (150-250 msec poststimulus).

3. The duration of the conductance increases during 1-IPSPs displayed a significant voltage dependence, decreasing as the membrane potential was depolarized and increasing with hyperpolarization.

4. The reversal potential of 1-IPSPs is significantly altered by reductions in the extracellular potassium concentration. Therefore it is concluded that 1-IPSPs in rat neocortical neurons are generated by the activation of a potassium conductance.

5. 1-IPSPs exhibit stimulation fatigue. Stimulation with a frequency of $1 \mathrm{~Hz}$ produces a complete fatigue of the conductance increases during 1-IPSPs after approximately 20 consecutive stimuli. Recovery from this fatigue requires minutes.

6. 1-IPSPs are not blocked by bicuculline but are blocked by baclofen.

\footnotetext{
${ }^{1}$ Clinical Neuropharmacology, Max Planck Institute for Psychiatry, Kraepelinstrasse 2, 8000 Munich 40, F.R.G.

${ }^{2}$ Present address: Department of Pharmacology, University College London, Gower Street, London WC1E 6BT, U.K.
} 


\section{INTRODUCTION}

We have been investigating $\gamma$-aminobutyric acid (GABA)-mediated inhibition in the rat frontal neocortex with intracellular recordings from an in vitro slice preparation of this structure. We have noted previously that electrical stimulation of superficial cortical layers evokes inhibitory postsynaptic potentials (IPSPs) in neurons (predominantly pyramidal cells) recorded in cortical layers 2 and 3 and that these IPSPs result from activation of $\mathrm{GABA}_{\mathrm{A}}$ receptors (Sutor and Zieglgänsberger, 1984; Sutor, 1986). It was shown: (1) that these IPSPs, which have times to peak amplitude of $20-25 \mathrm{msec}$ poststimulus and are referred to here as fast (f-IPSPs), have reversal potentials $\left(E_{\text {IPSP }}\right)$ that are very similar to the reversal potentials determined in the same neurons for membrane potential $\left(E_{\mathrm{m}}\right)$ changes produced by iontophoretic applications of GABA or the $\mathrm{GABA}_{\mathrm{A}}$ receptor agonist muscimol; (2) that reversal potential values for f-IPSPs and for responses to GABA or muscimol are similarly dependent on the transmembrane chloride ion gradient; and (3) that f-IPSPs and responses to GABA or muscimol were blocked by similar concentrations of the $\mathrm{GABA}_{\mathrm{A}}$ receptor antagonists bicuculline and picrotoxin. In addition, we have shown that the putative $\mathrm{GABA}_{B}$ receptor agonist baclofen has multiple actions on neurons in cortical layers 2 and 3 , increasing their conductance to potassium ions and depressing stimulation-evoked postsynaptic potentials by a different, presumably presynaptic, mechanism (Howe et al., 1987).

During the course of these studies, it became apparent that, in some neocortical neurons, IPSPs evoked by stimulation of superficial cortical layers consisted of two components. The second component had poststimulus times to peak amplitude of $150-250 \mathrm{msec}$ and its duration could approach $1 \mathrm{sec}$. This component reversed in polarity at $E_{\mathrm{m}}$ values approximately $15 \mathrm{mV}$ negative to the $E_{\text {IPSP }}$ values for f-IPSPs (measured 20-25 msec poststimulus) and appeared to be more prominent in neurons recorded during experiments in which the extracellular concentration of potassium was lowered. As such, it appeared that in these neurons the IPSP sequence consisted of $\mathrm{GABA}_{\mathrm{A}}$ receptor-mediated f-IPSPs which overlapped with and were followed by longduration IPSPs (1-IPSPs). These 1-IPSPs were at least superficially similar to longduration IPSPs recorded from neurons in other regions of the mammalian CNS, namely, CA1 and CA3 hippocampal pyramidal neurons (Fujita, 1979; Alger, 1984; Knowles et al., 1984; Newberry and Nicoll, 1984; Thalmann, 1984), hippocampal granule neurons (Thalmann and Ayala, 1982), neurons in the olfactory cortex (Constanti et al., 1980; Galvan et al., 1982; Satou et al., 1982), and neurons in the sensorimotor cortex (Connors et al., 1982). Further characterization of l-IPSPs evoked in neocortical neurons was of particular interest to us, because similar long-duration IPSPs evoked in hippocampal CA1 neurons were suggested to be secondary to activation of $\mathrm{GABA}_{\mathrm{B}}$ receptors. In this report, we describe the characteristics of the 1-IPSPs we record from neurons in the rat frontal neocortex.

\section{METHODS}

Adult Sprague-Dawley rats $(120-160 \mathrm{~g})$ were anesthetized with ether and decapitated. Coronal slices (nominally $500 \mu \mathrm{m}$ thick) were cut from the frontal poles of 
each cerebral cortical hemisphere. After incubation at room temperature for at least $1 \mathrm{hr}$, the slices were transferred to a recording chamber. The slices lay on a net and were superfused continuously $(4-5 \mathrm{ml} / \mathrm{min})$ with medium maintained at $36-37^{\circ} \mathrm{C}$. Warm humified air was circulated over the slices to prevent them from drying. The standard medium had the following composition $(\mathrm{m} M)$ : NaCl, $122.75 ; \mathrm{KCl}, 5.00 ; \mathrm{NaH}_{2} \mathrm{PO}_{4}$, $1.25 ; \mathrm{CaCl}_{2}, 2.50 ; \mathrm{MgSO}_{4}, 1.30 ; \mathrm{NaHCO}_{3}, 26$; and glucose, 10 (gassed continuously with $95 \% \mathrm{O}_{2} / 5 \% \mathrm{CO}_{2}$; final $\mathrm{pH}, 7.4$ ). Reductions in the $\mathrm{KCl}$ concentration of the medium were compensated for with equimolar $\mathrm{NaCl}$. Bicuculline methiodide (Sigma) and baclofen (Ciba-Geigy) were applied in the superfusion medium. Steady-state responses to changes in the ionic composition of the medium or to the application of drugs were achieved after approximately $5 \mathrm{~min}$ of continuous superfusion.

Intracellular recordings were made from neurons in layers 2 and 3 of the dorsomedial frontal neocortex with glass microelectrodes that were filled with either potassium acetate $(4 M, \mathrm{pH} 7.0)$ or $2 M$ potassium methylsulfate. Postsynaptic potentials were evoked by electrical stimulation $(50 \mu \mathrm{sec}$ in duration) of superficial cortical layers with a bipolar silver stimulation electrode (insulated to within $250 \mu \mathrm{m}$ of the tips; tip diameter, $100 \mu \mathrm{m}$; tip separation, $250 \mu \mathrm{m}$ ). The stimulation electrode was positioned such that a line drawn between the tips would be roughly perpendicular to the dorsal surface of the cortex and so that the dorsalmost tip of the electrode was $100-150 \mu \mathrm{m}$ from this surface. The stimulation electrode was placed 0.8 to $1.5 \mathrm{~mm}$ from the medial surface of the slice and all recordings were made at positions $0.5-1.0 \mathrm{~mm}$ medial to the stimulation electrode. Current was injected via a Dagan 8100 amplifier equipped with a bridge circuit and also with a sample and hold current-clamp mode (switching frequency, $1-2 \mathrm{kHz} ; 25 \%$ duty cycle). When recordings were made in the switched current-clamp mode, the headstage output was displayed continuously on a separate oscilloscope and the capacity compensation and switching frequency adjusted so that the voltage transients in response to rectangular current injections were as rectangular as possible and had decayed completely before the voltage was measured. Neuronal input resistance $\left(R_{\mathrm{N}}\right)$ was determined for each neuron at resting membrane potential $\left(E_{\mathrm{m}}\right)$ with injections of 150 -msec rectangular current steps that produced hyperpolarizing voltage transients of $\leqslant 10 \mathrm{mV}$ or from the slope of current-voltage plots. In addition, estimates of $R_{\mathrm{N}}$ were made with shorter current injections of larger magnitudes before and during IPSPs to estimate the relative conductance increases during these IPSPs.

IPSPs were measured at their peak amplitudes. For f-IPSPs, these times to peak (20-30 msec poststimulus) were independent of $E_{\mathrm{m}}$. For l-IPSPs, however, the times to peak were dependent on the $E_{\mathrm{m}}$ value at which the stimuli were applied (see Results). At $E_{\mathrm{m}}$ values positive to approximately $-55 \mathrm{mV}$, there was often no clear separation between f-IPSPs and 1-IPSPs, and as such, assignment of a time to peak value became arbitrary. In these cases, 1-IPSP amplitudes were measured at the shortest time to peak value determined for IPSP sequences for which the decay was clearly biphasic, and these amplitudes used for the construction of plots to determine $E_{\mathrm{IPSP}}$ values. At $E_{\mathrm{m}}$ values at which both f-IPSPs and 1-IPSPs were depolarizing, there was also no clear separation between the two IPSPs on the voltage trace; and depolarizing 1-IPSPs were measured at the longest poststimulus time to peak determined for hyperpolarizing 1-IPSPs evoked in the same neuron. 
Membrane potential values were corrected for offset potentials by rapidly withdrawing the electrode at the end of the recording. All values are reported as the mean \pm SD. Two-tailed Student's $t$ tests were used for statistical comparisons of two sample means.

\section{RESULTS}

Intracellular recordings were obtained from 38 neurons in cortical layers 2 and 3 in which 1-IPSPs could be evoked and studied clearly. These 38 neurons had the following characteristics: resting $E_{\mathrm{m}},-80 \pm 9 \mathrm{mV}$; resting $R_{\mathrm{N}}, 25 \pm 11 \mathrm{M} \Omega$; action potential amplitudes, $109 \pm 12 \mathrm{mV}$; and action potential overshoots, $30 \pm 6 \mathrm{mV}$. Current-voltage relationships of the neurons were nonlinear and displayed inward rectification. On average, the $R_{\mathrm{N}}$ measured at an $E_{\mathrm{m}}$ value of $-65 \mathrm{mV}$ was about twice that measured at $-95 \mathrm{mV}$. These characteristics are comparable to the characteristics of other rat neocortical neurons recorded in our laboratory (see Howe et al., 1987; Sutor, 1986), and there were no apparent differences in these properties or in the discharge behavior of neocortical neurons in which stimulation-evoked 1-IPSPs were recorded and those in which stimulation of superficial cortical layers did not evoke 1-IPSPs. In other studies employing intracellular injections of lucifer yellow, 22 of 25 neurons stained under experimental conditions similar to those reported here were identified as pyramidal cells (Sutor, 1986). These results and the large size of cortical pyramidal cells suggest that the majority of the 38 neurons reported on here were pyramidal neurons, although we have not verified this directly for the present sample.

\section{Dependence of I-IPSPs on Stimulus Intensity}

Stimulation-evoked potentials were recruited in the following characteristic sequence as the stimulus intensity was increased: excitatory postsynaptic potentials (EPSPs) with times to peak of $7-10 \mathrm{msec}$ poststimulus; f-IPSPs; 1-IPSPs; action potentials. Figure 1A depicts the recruitment sequence of postsynaptic potentials at stimulation intensities subthreshold for the orthodromic evocation of action potentials for one neuron. Because of the high resting membrane potentials of rat neocortical neurons, 1-IPSPs which were quite prominent when stimuli were applied shortly after impalement, or after depolarizing the neuron with current injection, were sometimes not apparent when the stimuli were applied at resting $E_{\mathrm{m}}$ (compare 20-V stimuli in Fig. 1B). Thus accurate determination of the threshold stimulation intensity for evocation of 1-IPSPs usually required that stimuli were also applied after depolarizing the neuron with current injections (Fig. 1A). In 14 of 19 neurons in which careful determinations of the threshold stimulation intensity for 1-IPSPs were performed, this threshold was less than the stimulation threshold intensity for evocation of action potentials. The mean stimulation intensity required to produce 1-IPSPs in these 19 cells was $0.86 T$, where $T$ is the action potential stimulation threshold. As the stimulation intensity was increased, the amplitude and duration of 1-IPSPs also increased (Figs. 1A and B).

Stimulation intensities that evoked 1-IPSPs also always evoked EPSPs and usually f-IPSPs (mean thresholds, $0.58 T$ and $0.78 T ; N=34$ and 24, respectively). Thus the presence of preceding EPSPs and f-IPSPs precluded a precise determination of the latency to onset of 1-IPSPs. In a few neurons, it appeared that significant 
A

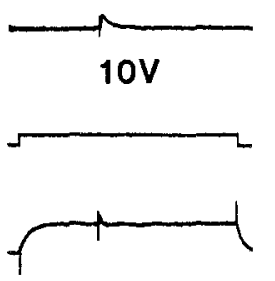

B
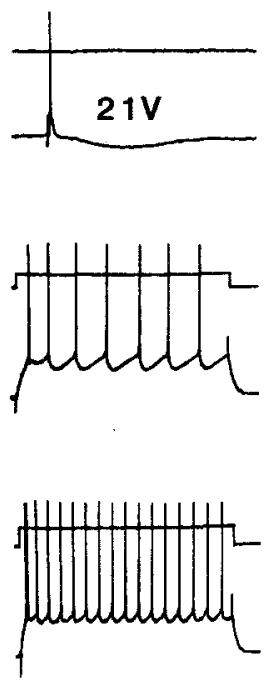
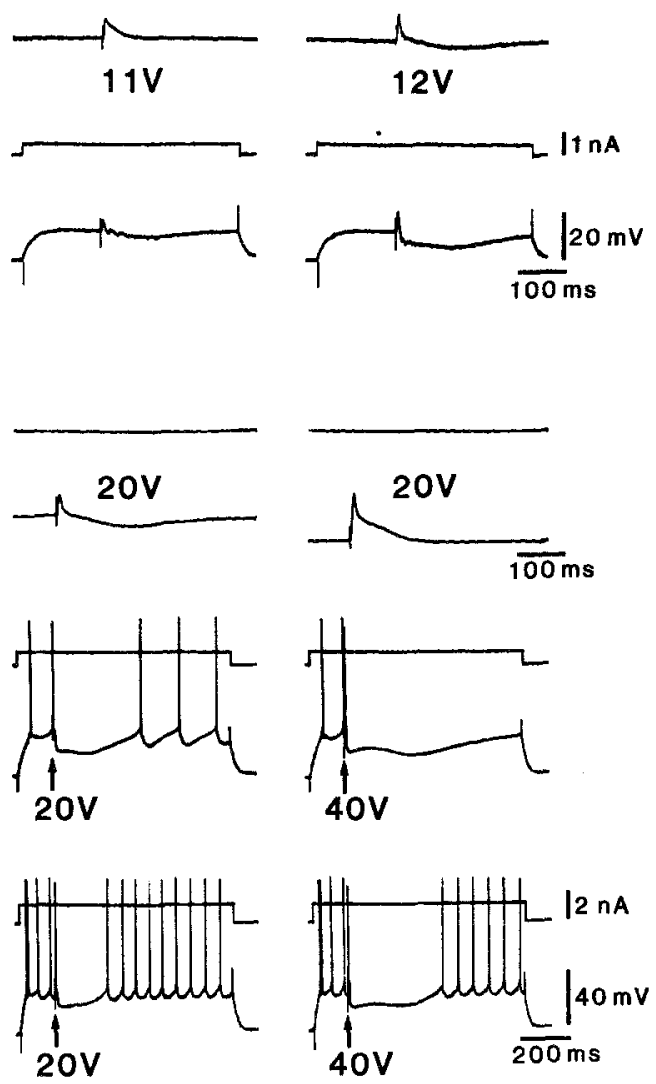

Fig. 1. 1-IPSPs in rat neocortical neurons in vitro. (A) Top row: Stimulation-evoked postsynaptic potential sequences evoked with subthreshold stimuli $(0.125 \mathrm{~Hz})$ of increasing intensities at resting $E_{\mathrm{m}}$ $(-82 \mathrm{mV})$. Stimulation with $12 \mathrm{~V}$ evokes a hyperpolarizing potential with a time-to-peak amplitude of approximately $120 \mathrm{msec}$. Second row: Postsynaptic potential sequences evoked after depolarizing $E_{\mathrm{m}}$ with rectangular current injections. The long-duration hyperpolarizing potential, which was only apparent with $12-\mathrm{V}$ stimulation at resting $E_{\mathrm{m}}$, is evident at $11 \mathrm{~V}$ when the stimuli are applied during the current injections, and $12 \mathrm{~V}$ evokes a biphasic hyperpolarizing potential (f-IPSP/1-IPSP). (B) Recording from another neuron. Top row: Action potential (left) and postsynaptic potential sequences (middle) evoked with $21-\mathrm{V}$ and $20-\mathrm{V}$ stimulation, respectively, shortly after impalement $\left(E_{\mathrm{m}}=-68 \mathrm{mV}\right.$ ) and with $20-\mathrm{V}$ stimulation $15 \mathrm{~min}$ later (resting $E_{\mathrm{m}}=-88 \mathrm{mV}$ ). Note that the 1IPSPs, which are quite prominent when the stimuli are applied at $E_{\mathrm{m}}=-68 \mathrm{mV}$, are not readily apparent after the neuron has reestablished its stable resting $E_{\mathrm{m}}$ (right). Note also that the amplitude and duration of I-IPSPs evoked with $21-\mathrm{V}$ or with $20-\mathrm{V}$ stimuli are very similar but that the presence of an action potential $(21 \mathrm{~V})$ shifts the potential sequence at the peak amplitude of f-IPSPs $(20-25 \mathrm{msec}$ poststimulus) approximately $3 \mathrm{mV}$ in the hyperpolarized direction. Second and third rows: Trains of action potentials were evoked with 850 -msec depolarizing current steps (left, 1.0 or $1.4 \mathrm{nA}$ ), and a $20-\mathrm{V}$ (middle) or 40-V (right) stimulus was then applied during these trains. Increasing the stimulus intensity from 20 to $40 \mathrm{~V}$ approximately doubles the duration of the stimulation-evoked inhibition. Note that with $40-\mathrm{V}$ stimulation (second row, right) the peak amplitudes of hyperpolarizing f-IPSPs and 1-IPSPs are separated by a transient depolarization, suggesting that activation of I-IPSPs which is significant enough to overcome the decay of f-IPSPs does not occur until almost 100 msec poststimulus in this neuron. Note also that, although the peak amplitude of l-IPSPs is larger than that of f-IPSPs when the stimuli are applied during the spike trains evoked with $1.0 \mathrm{nA}$ (second row), the duration of the inhibition and the apparent amplitude of 1-IPSPs are significantly reduced when the magnitude of the depolarizing current step is increased to $1.4 \mathrm{nA}$. In this and all subsequent figures, the current monitor was set such that zero current corresponds to the zero potential. 
activation of 1-IPSPs did not occur until approximately $50 \mathrm{msec}$ poststimulus, because the peaks of hyperpolarizing f-IPSPs and 1-IPSPs were separated by a transient depolarization (Fig. 1B). These depolarizations were not subthreshold EPSPs. They never generated action potentials when the neurons were depolarized with current injections, and they were clearly different from the late EPSPs we have described before (Howe et al., 1987). Rather, they appeared to represent the initial decay of preceding f-IPSPs. In most neurons, however, hyperpolarizing f-IPSPs and 1-IPSPs were separated only by a shoulder on the potential sequence $25-35 \mathrm{msec}$ poststimulus.

Although 1-IPSPs were evoked with stimulation intensities subthreshold for evocation of action potentials, in order to increase the amplitudes of 1-IPSPs and enable them to be studied more easily, they were evoked in most experiments with stimulation intensities that were suprathreshold for action potential generation. It should be emphasized, however, that the presence of preceding action potentials did not appear to influence significantly the characteristics of 1-IPSPs. Thus, 1-IPSPs evoked with stimulation intensities just subthreshold for action potential generation and those evoked at action potential threshold intensities were very similar in peak amplitude, times to peak, and duration (Fig. 1B, compare left and middle traces in top row). Hyperpolarizing potentials that followed short depolarizations or action potentials produced by direct current injections were lower in amplitude and had shorter times to peak and durations than 1-IPSPs. Thus 1-IPSPs are not a consequence of preceding depolarizing potentials and can be clearly distinguished from the spike afterhyperpolarization (compare also the kinetics of spike afterhyperpolarizations and 1-IPSPs in the last two rows of traces in Fig. 1B). The presence of preceding action potentials did appear to influence slightly the early portion of the IPSP sequence and cause a 1- to 3-mV hyperpolarizing shift in the amplitude of the IPSP sequence measured 20-25 msec poststimulus, i.e., at the peak of f-IPSPs (Fig. 1B, top row, left and middle traces).

Increases in membrane conductance were measured during 1-IPSPs and increased in amplitude as the stimulation intensity was increased. Conductance increases large enough to block a directly evoked action potential could often be measured $150-250 \mathrm{msec}$ poststimulus at stimulus intensities below those at which 1-IPSPs became frankly apparent on the voltage trace, suggesting that 1-IPSPs were evoked by these former intensities but were too low in amplitude to be distinguished from the background noise of the recording system. The conductance increases measured during the peak of 1-IPSPs evoked with stimulation intensities suprathreshold for action potential generation could be quite large, corresponding to up to $60 \%$ decreases in $R_{\mathrm{N}}$. The conductance increases determined at the peak amplitude of 1-IPSPs were always, however, less than the conductance increases determined at the peak amplitude of the preceding f-IPSPs that were evoked in the same neuron with the same stimulus intensity. Furthermore, the inhibitory effect of 1-IPSPs on directly evoked action potential discharge (Fig. 1B) could be overridden by increasing the magnitude of the depolarizing current and, consequently, the frequency of the action potential discharge, whereas the inhibition produced by f-IPSPs was much less sensitive to the frequency of the action potential discharge during which they were evoked and remained effective even at the highest firing rates it was possible to produce within the current passing limits of the electrodes. 


\section{l-IPSPs Are Potassium Dependent}

Determinations of the reversal potential of each type of IPSP were made for 18 and 20 neurons in experiments where the concentration of potassium in the medium was 5.00 and $2.50 \mathrm{~m} M$, respectively. For 10 neurons recorded in $5.00 \mathrm{~m} M$ potassium medium and 10 neurons recorded in $2.50 \mathrm{mM}$ potassium medium (where for each of the 20 neurons, l-IPSPs were evoked with $2 T$ stimulation for $E_{\mathrm{IPSP}}$ determinations), the mean $E_{\mathrm{IPSP}}$ values for 1-IPSPs were $-81 \pm 4$ and $-91 \pm 4 \mathrm{mV}$, respectively. These values were significantly different from each other $\left(P<0.00005\right.$; individual $E_{\mathrm{IPSP}}$ values obtained from least-squares linear regression analyses of five to nine point plots for each neuron). A similar conclusion regarding the significance of the dependence of $E_{\text {IPSP }}$ values for 1-IPSPs on the extracellular potassium concentration was arrived at when one linear regression analysis was performed on each set of data and the $E_{\mathrm{IPSP}}$ values determined for each set were compared with an ANOVA (Fig. 2). The mean $E_{\mathrm{f}-\mathrm{IPSP}}$ values determined in 5.00 and $2.50 \mathrm{mM}$ potassium were $-68 \pm 3$ and $-72 \pm 2 \mathrm{mV}(N=10$ neurons each; significantly different from each other, $P<0.01)$.

During recordings from five additional neurons, in each neuron the $E_{\mathrm{IPSP}}$ value of 1-IPSPs was determined in $5.00 \mathrm{~m} M$ and also in $1.25 \mathrm{~m} M$ potassium medium (Fig. 3). Reversal potential values for 1-IPSPs were shifted $17 \pm 4 \mathrm{mV}$ in the hyperpolarized direction when the extracellular potassium concentration was switched from 5.00 to
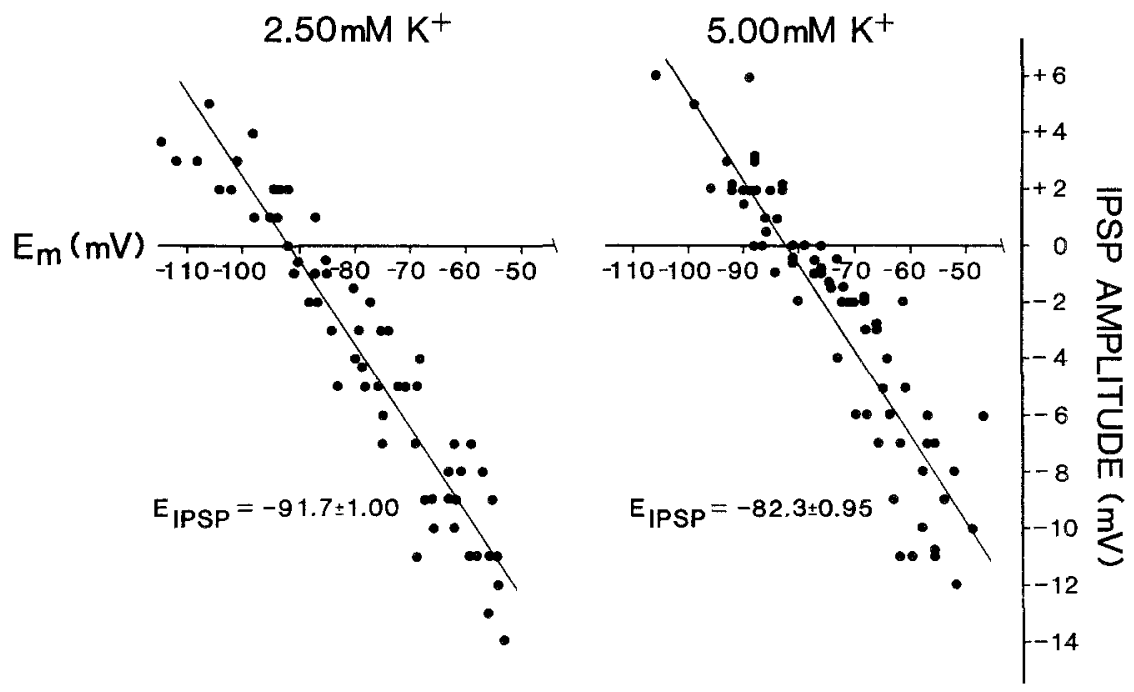

Fig. 2. The reversal potential of l-IPSPs is potassium dependent. Plots of the peak amplitude of l-IPSPs as a function of $E_{\mathrm{m}}$ from measurements made on 10 neurons recorded in $2.50 \mathrm{mM}$ potassium medium (left) and on 10 other neurons recorded in $5.00 \mathrm{~m} M$ potassium medium (right). 1-IPSPs were evoked with $2 T$ stimulation intensity for each of the 20 neurons. A linear regression analysis was performed on each set of data and the expected $E_{\mathrm{m}}$ values at zero IPSP amplitude were calculated from these analyses ( $E_{I P S P} \pm S D$ as indicated in plots). Comparison of the two $E_{\text {IPSP }}$ values estimated from these analyses indicates that they are significantly different from each other $(P<0.00005)$. Comparison of the mean $E_{\text {IPSP }}$ values calculated for each group of neurons from individual $E_{\mathrm{IPSP}}$ estimates for each neuron in each group also indicates that the

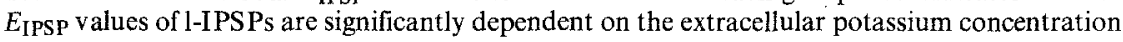
(see text). 

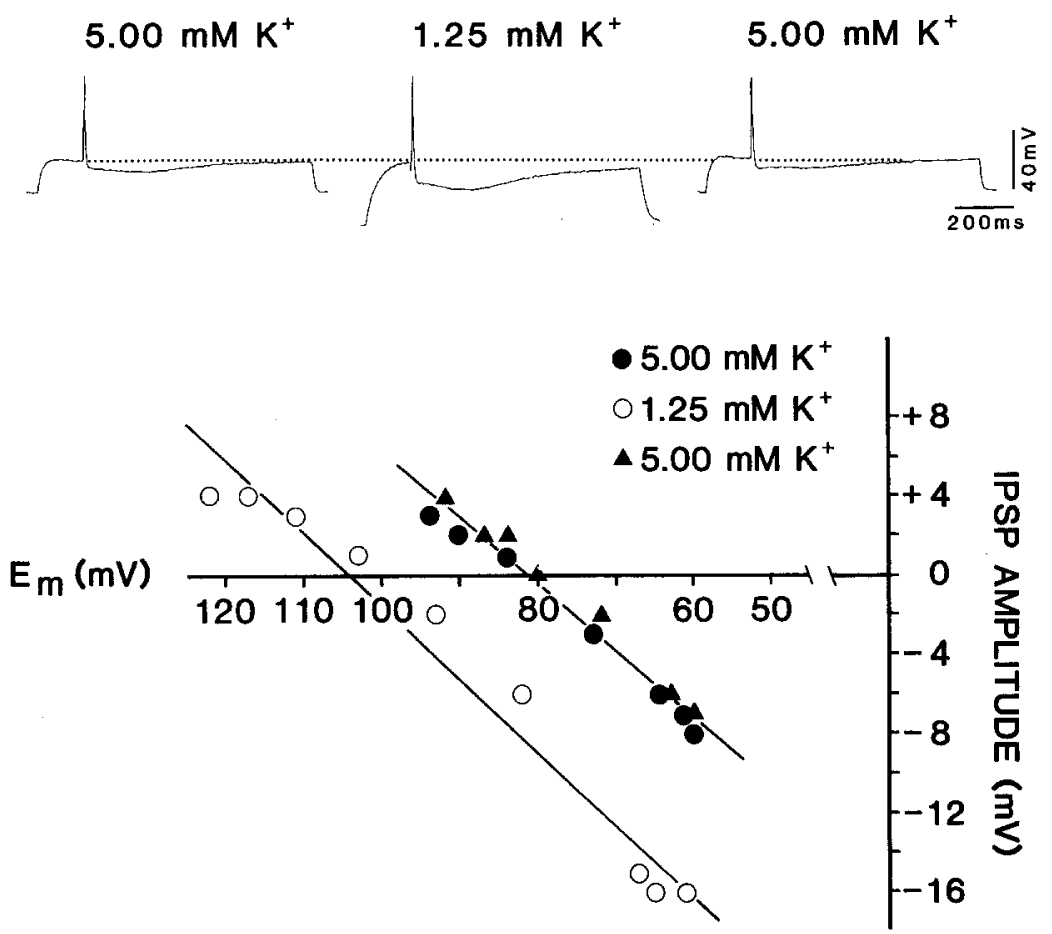

Fig. 3. The reversal potential of 1-IPSPs is significantly altered by alterations of the extracellular potassium concentration. Top: Postsynaptic potential sequences evoked with $2 T$ stimulation after shifting $E_{\mathrm{m}}$ with 900 -msec depolarizing current injections $(0.90$, 0.65 , and $0.80 \mathrm{nA}$, left to right) in $5.00 \mathrm{mM}$ potassium medium, $20 \mathrm{~min}$ after switching to $1.25 \mathrm{mM}$ potassium medium, and $20 \mathrm{~min}$ after returning to $5.00 \mathrm{~m} M$ potassium (traces were digitized, filtered at $500 \mathrm{~Hz}$, and plotted on an $x-y$ plotter). The dotted line represents $E_{\mathrm{m}}=-64 \mathrm{mV}$. Bottom: Plots of each complete set of data obtained for determinations of $E_{\text {IPSP. }}$. Changing the potassium concentration of the medium from 5.00 to $1.25 \mathrm{mM}$ shifts the $E_{\mathrm{IPSP}}$ from -82 to $-104 \mathrm{mV}$. Resting $E_{\mathrm{m}}$ was -84 and $-104 \mathrm{mV}$ in 5.00 and $1.25 \mathrm{~m} M$ potassium, respectively. Stimulation frequency was $0.125 \mathrm{~Hz}$.

$1.25 \mathrm{~m} M(N=5$; significantly different from $0, P<0.001)$. Altering the potassium concentration from 5.00 to $1.25 \mathrm{mM}$ shifted the $E_{\text {IPSP }}$ for f-IPSPs in these same five neurons $-4 \pm 2 \mathrm{mV}$. The latter difference was also significantly different from zero $(P<0.05)$; however, comparison of the magnitudes of the changes in $E_{\mathrm{IPSP}}$ values indicates clearly that the $E_{\mathrm{IPSP}}$ values of f-IPSPs were less sensitive to changes in the extracellular potassium concentration than were those of 1-IPSPs.

The differential sensitivity of f-IPSPs and 1-IPSPs to changes in the transmembrane gradient of potassium ions is also indicated by comparison of the mean values determined in different extracellular concentrations of potassium for the difference between the two $E_{\mathrm{IPSP}}$ values. 1-IPSPs reversed in polarity at a $E_{\mathrm{m}}$ value $12 \pm 2 \mathrm{mV}$ negative to the $E_{\mathrm{IPSP}}$ for f-IPSPs in $5.00 \mathrm{mM}$ potassium medium and $18 \pm 4 \mathrm{mV}$ negative to the $E_{\text {IPSP }}$ for f-IPSPs in $2.50 \mathrm{~m} M$ potassium $(N=10$ for each concentration; values significantly different from each other, $P<0.0005)$. The mean differences between the two $E_{\mathrm{IPSP}}$ values were $13 \pm 2 \mathrm{mV}$ in $5.00 \mathrm{mM}$ potassium 
medium and $24 \pm 3 \mathrm{mV}$ in $1.25 \mathrm{mM}$ potassium medium for the five neurons in which the determinations were made in each potassium concentration (significantly different from each other, $P<0.0005$ ).

The reversal potentials of 1-IPSPs and of the hyperpolarizations that follow a train of directly evoked action potentials were compared in seven neurons. The mean $E_{\text {IPSP }}$ value determined for 1-IPSPs did not differ significantly from the mean reversal potential determined for spike train afterhyperpolarizations, which in rat neocortical and other cortical neurons are secondary to an increase in potassium conductance (Hotson et al., 1980; Schwartzkroin and Stafstrom, 1980; Sutor, 1986).

\section{The Durations of 1-IPSPs and of Their Associated Conductance Increases Exhibit an Apparent Voltage Dependence}

The duration of hyperpolarizing 1-IPSPs decreased as they were evoked at more depolarized $E_{\mathrm{m}}$ values. If stimuli were applied at $E_{\mathrm{m}}$ values positive to $-55 \mathrm{mV}$, 1-IPSPs could sometimes no longer be detected, i.e., the potential decayed smoothly and monophasically from the peak of f-IPSPs. At the high resting $E_{\mathrm{m}}$ values of neurons in the rat neocortical slice, however, the duration of l-IPSPs could approach $1 \mathrm{sec}$. The duration of 1-IPSPs evoked at resting $E_{\mathrm{m}}$ with stimulation intensities suprathreshold for action potential generation were determined in eight neurons by measuring the duration of the poststimulus blockade of a directly evoked action potential, as depicted in Fig. 4. The mean duration was $835 \pm 190 \mathrm{msec}$, with a longest duration of $1200 \mathrm{msec}$ poststimulus.

In four neurons, we used a similar protocol to estimate the duration of the conductance increases during IPSP sequences evoked both at resting $E_{\mathrm{m}}$ and after shifting $E_{\mathrm{m}}$ to different values with constant current injections. For each neuron, the duration of the conductance increases associated with l-IPSPs (taken as the duration of the poststimulus block of a directly evoked action potential) was clearly voltage dependent, decreasing as $E_{\mathrm{m}}$ was depolarized and increasing as $E_{\mathrm{m}}$ was hyperpolarized (Fig. 4). For two of these neurons, this result was verified more than twice. At early poststimulus times, however, the relative magnitude of the conductance increases during the IPSP sequences appeared to increase as $E_{\mathrm{m}}$ was depolarized, and the magnitude of the relative conductance increases measured between 200 and $400 \mathrm{msec}$ poststimulus appeared to be relatively independent of the $E_{\mathrm{m}}$ value at which the stimuli were applied (Fig. 4B). Thus, whereas the duration of the conductance increases associated with 1-IPSPs exhibit a quite steep voltage dependence, shortening approximately $150 \mathrm{msec}$ for a $10-\mathrm{mV}$ depolarization, the conductance increases at the peak amplitudes of 1-IPSPs appeared to be voltage independent.

\section{The Time to Peak Amplitude of I-IPSPs Is Voltage and Potassium Dependent}

The poststimulus time at which the peak amplitude of hyperpolarizing l-IPSPs occurred was clearly dependent on the $E_{\mathrm{m}}$ value at which the l-IPSPs were evoked for each of the 38 neurons. This time to peak amplitude decreased as $E_{\mathrm{m}}$ was depolarized and increased with hyperpolarization (Fig. 5). The time to peak amplitude of hyperpolarizing 1-IPSPs was taken as the time at which the most negative $E_{\mathrm{m}}$ value was attained after the shoulder on the IPSP sequence. This value was either estimated 

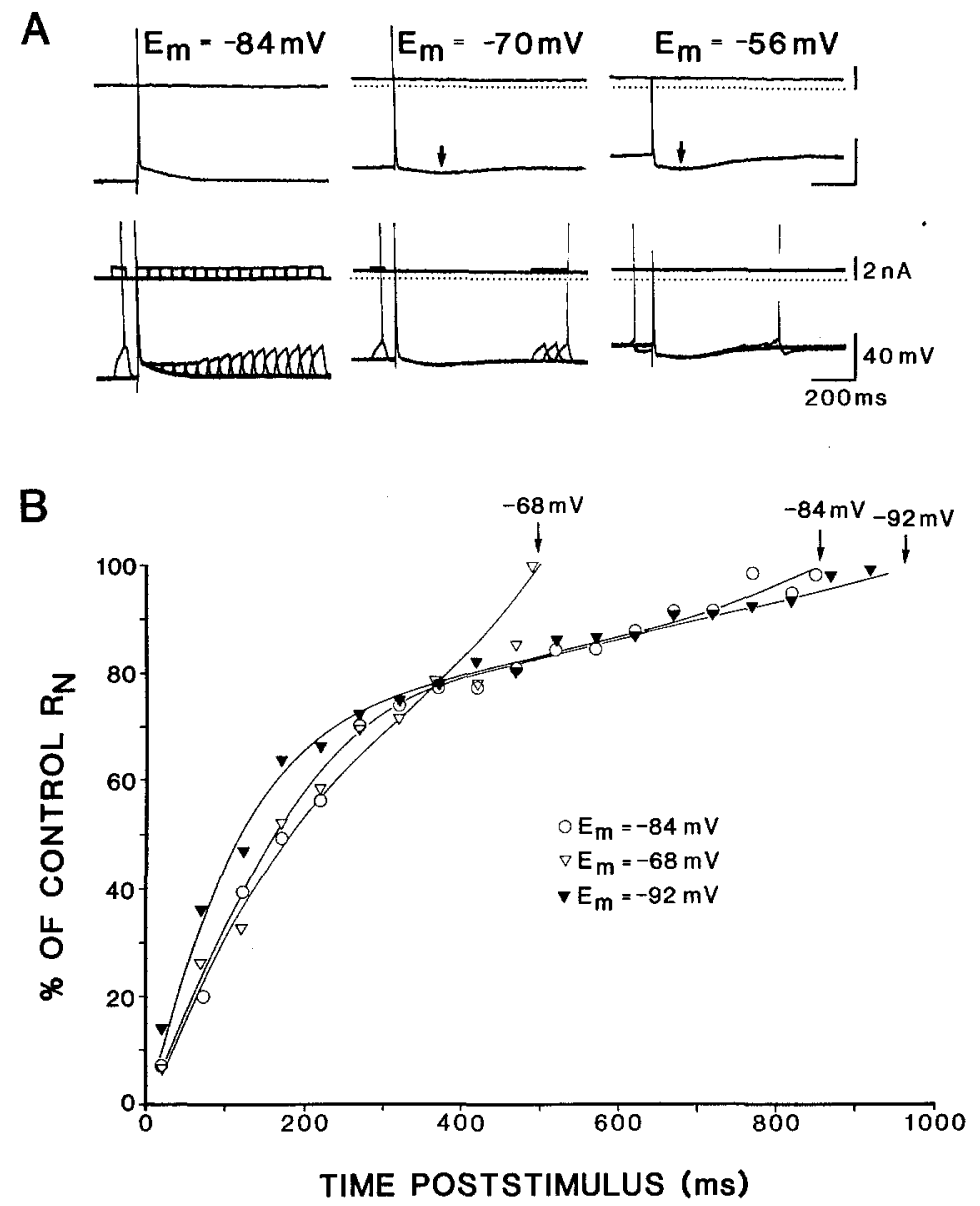

Fig. 4. The duration of the conductance increases during l-IPSPs is voltage dependent. (A) Top row, left: Action potential and postsynaptic potential sequence evoked with $2 T$ stimulation $(0.125 \mathrm{~Hz})$ at resting $E_{\mathrm{m}}$ $(-84 \mathrm{mV}$, bridge mode; other recordings are in switched current-clamp mode $(1 \mathrm{kHz})$ and some spikes are cut). Depolarizing $E_{\mathrm{m}}$ (middle and right; zero-current line indicated by dashed line) reveals the presence of a 1-IPSP (arrows). Second row: At each $E_{\mathrm{m}}$ value, a $60-\mathrm{msec}$ current step of the magnitude just necessary to evoke an action potential prestimulus was applied poststimulus at 50-msec intervals until the current step evoked an action potential. That the current step did evoke an action potential in the absence of a stimulus was verified every third poststimulus time. The action potential was blocked until $910 \mathrm{msec}$ at $E_{\mathrm{m}}=-84 \mathrm{mV}$ (left; complete measurement sequence shown to $750 \mathrm{msec}$ ), $700 \mathrm{msec}$ at $E_{\mathrm{m}}=-70 \mathrm{mV}$ (middle; current steps at 600,650 , and $700 \mathrm{msec}$ shown), and $500 \mathrm{msec}$ at $E_{\mathrm{m}}=-56 \mathrm{mV}$ (right; current steps are $+0.05 \mathrm{nA}$ in magnitude and are obscured by the noise in the current trace). (B) A relative membrane resistance $\left(R_{N}\right)$ was determined at various poststimulus times for another neuron with an experimental protocol similar to that in $\mathrm{A}$. These values were expressed as percentage control $R_{\mathrm{N}}$ and are plotted vs poststimulus time for three different $E_{\mathrm{m}}$ values (arrows indicate the poststimulus time at which the action potential was not blocked; control $R_{\mathrm{N}}$ estimated separately for each $E_{\mathrm{m}}$ ). The duration of the poststimulus block of the directly evoked action potential shortens as $E_{\mathrm{m}}$ is depolarized from resting $E_{\mathrm{m}}(-84 \mathrm{mV})$ and lengthens as $E_{\mathrm{m}}$ is hyperpolarized. The action potential was no longer blocked at 490,870 , and $970 \mathrm{msec}$ poststimulus if the stimuli $(0.125 \mathrm{~Hz})$ were applied at $E_{\mathrm{m}}$ values of $-68,-84$, and $-92 \mathrm{mV}$, respectively. The relative conductances during the early portion of the IPSP sequence appear to increase with depolarization, however, and the relative conductance increases between 200 and $400 \mathrm{msec}$ poststimulus are essentially the same for each of the $E_{\mathrm{m}}$ values tested. 


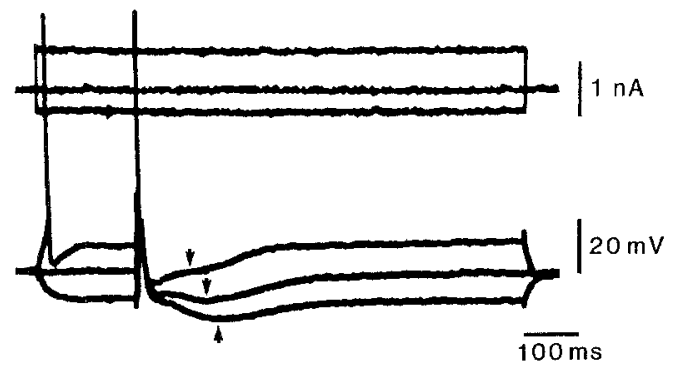

Fig. 5. The poststimulus time at which the peak amplitude of 1-IPSPs occurs is voltage dependent. Top: Action potentials and postsynaptic potential sequences evoked with $2 T$ stimulation at resting $E_{\mathrm{m}}$ and after shifting $E_{\mathrm{m}}$ with 900 -msec current injections $(+0.80$ and $-0.35 \mathrm{nA})$. The poststimulus time-topeak amplitude of 1-IPSPs (arrows) is shortened when $E_{\mathrm{m}}$ is depolarized and lengthened when $E_{\mathrm{m}}$ is hyperpolarized. Bottom: Plot of the poststimulus time-to-peak 1-IPSP amplitude vs the $E_{\mathrm{m}}$ at which the stimuli were applied from the complete set of data obtained from the neuron depicted above. The time-to-peak amplitude of hyperpolarizing 1-IPSPs is a linear function of $E_{\mathrm{m}}$ between -55 and $-95 \mathrm{mV}$ in this neuron. Resting $\quad E_{\mathrm{m}}=-66 \mathrm{mV} ; \quad E_{\mathrm{IPSP}}=-97 \mathrm{mV}$ (2.50 $\mathrm{mM}$ potassium); stimulation frequency, $0.125 \mathrm{~Hz}$.

by eye from the replayed analog signals or measured with a digital oscilloscope (Nicollet 3091) by moving a cursor along the digitized trace. Some signals were analyzed both ways, and the results obtained were essentially identical. If, instead, 1-IPSPs were always measured at the poststimulus time at which the peak amplitude occurred at resting $E_{\mathrm{m}}$, the resultant $E_{\mathrm{IPSP}}$ plots were curvilinear and hyperpolarizing amplitudes actually decreased as $E_{\mathrm{m}}$ was depolarized positive to $-60 \mathrm{mV}$ (cf. Satou et al., 1982; Alger, 1984; Thalmann, 1984).

As mentioned above, at $E_{\mathrm{m}}$ values positive to approximately $-55 \mathrm{mV}$ there was often no clear separation between f-IPSPs and 1-IPSPs, as was also the case when both f-IPSPs and 1-IPSPs were depolarizing. In these cases, the assignment of a time to peak amplitude was necessarily arbitrary. Between $E_{\mathrm{m}}$ values of -55 and $-85 \mathrm{mV}$, however, the peak amplitudes of the two types of IPSPs could be clearly distinguished and time-to-peak values could be estimated accurately, with allowance for the random fluctuations introduced by noise in the voltage trace. Over this voltage range, plots of the time to peak amplitude of l-IPSPs vs $E_{\mathrm{m}}$ were linear (Fig. 5). Regression analyses were performed on such plots for 22 neurons in which four to eight determinations were made between -55 and $-85 \mathrm{mV}$ (range and median of $r$ values for these analyses, $0.88-1.00$ and 0.97 ). The expected time-to-peak values for $E_{\mathrm{m}}$ values of -60 and $-80 \mathrm{mV}$ and the difference between these two expected values were calculated for 
each neuron from these analyses. The time-to-peak value was $70 \pm 34 \mathrm{msec}$ longer at $-60 \mathrm{mV}$ than it was at $-80 \mathrm{mV}$ (significantly different from $0, P<0.00005$ ).

These analyses also indicated that, at the same $E_{\mathrm{m}}$ value, the time-to-peak values of neurons in $2.50 \mathrm{mM}$ potassium were significantly shorter than the time-to-peak values of neurons recorded in $5.00 \mathrm{mM}$ potassium medium. This shortening of the time-to-peak I-IPSP amplitude with decreases in the extracellular potassium concentration was verified by similar analyses of the data from the five neurons that were recorded in both 5.00 and $1.25 \mathrm{mM}$ potassium.

\section{I-IPSPs Exhibit Stimulation Fatigue}

The amplitude of 1-IPSPs decreased with consecutive stimuli when these stimuli were applied at frequencies $>0.125 \mathrm{~Hz}$. This reduction was already clearly evident by the second stimulus. Our results indicate that the reduction of 1-IPSP amplitudes with continuing stimulation can be accounted for by a reduction of the conductance increases during l-IPSPs (Fig. 6A). The dependence of this stimulation fatigue on stimulation frequency was further investigated in four neurons. A stimulation frequency of $1 \mathrm{~Hz}$ was approximately the most effective at producing stimulation fatigue (complete fatigue with 15 to 20 stimuli). Increasing the stimulation frequency beyond $1 \mathrm{~Hz}$ resulted in a decrease in the extent of fatigue obtained with a given number of stimuli (Fig. 6B). Recovery from complete fatigue took 3 to $5 \mathrm{~min}$, and the time required for complete recovery did not increase as the stimulation frequency was increased beyond $2 \mathrm{~Hz}$ (Fig. 6C). The reduction in the amplitudes of f-IPSPs upon repetitive stimulation appeared to be due in part to a genuine use-dependent fatigue of the f-IPSP conductance but was also related to shifts in their $E_{\mathrm{IPSP}}$ values, as is evident in Fig. 6A, where the polarity of f-IPSPs reverses during the stimulus train, which is presumably the result of transmembrane chloride redistribution.

Stimulation at frequencies $\geqslant 2 \mathrm{~Hz}$ caused changes in resting $E_{\mathrm{m}}$, usually depolarizations, during the course of the stimulus trains. The extent of these changes increased with increasing frequency, but at a stimulation frequency of $2 \mathrm{~Hz}$ they never were more than 3 or $4 \mathrm{mV}$. These shifts in $E_{\mathrm{m}}$ occurred essentially immediately, i.e., from the first to the second or third stimulus, and $E_{\mathrm{m}}$ repolarized when the stimuli were discontinued (Fig. 6A). These shifts in $E_{\mathrm{m}}$ seemed to be due primarily to the application of the next stimulus before the IPSP sequence evoked by the preceding stimulus had decayed completely. These shifts were too small to influence the conductance estimates significantly, and the rapid onset and recovery of these shifts did not correlate with the time course of the stimulation fatigue we observed or of the recovery from this fatigue.

\section{l-IPSPs Are Bicuculline Resistant but Are Blocked by Baclofen}

Applications of bicuculline were made on four neurons in which 1-IPSPs were evoked by stimulation of superficial cortical layers. The concentrations of bicuculline applied $(10$ to $50 \mu M)$ led to very marked reductions in f-IPSPs and, consequently, resulted in significant increases in synaptic excitability which complicated the analysis of the effect of bicuculline on l-IPSPs. Despite this complication, however, during the approach to equilibration of the slice with bicuculline (application times $<5 \mathrm{~min}$ ), there were times in each of the four cases when f-IPSPs were very clearly and 

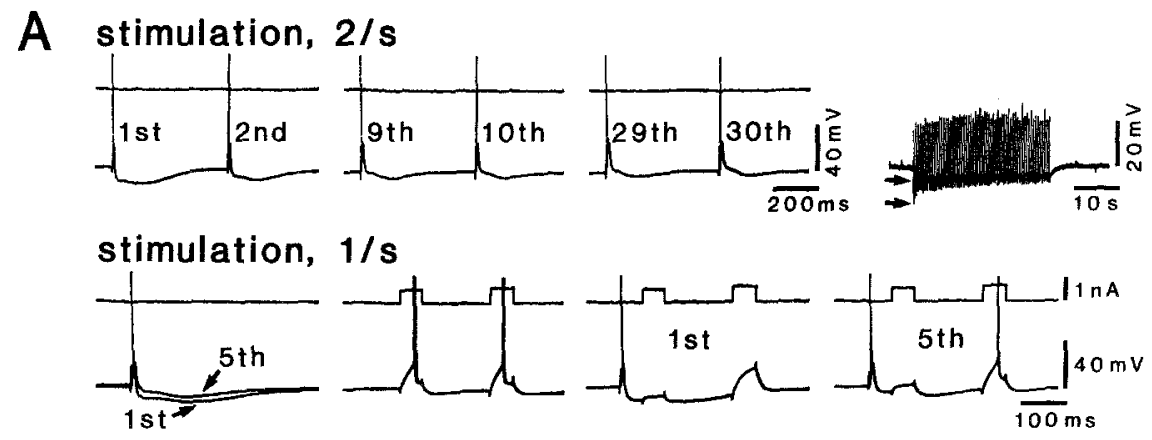

B

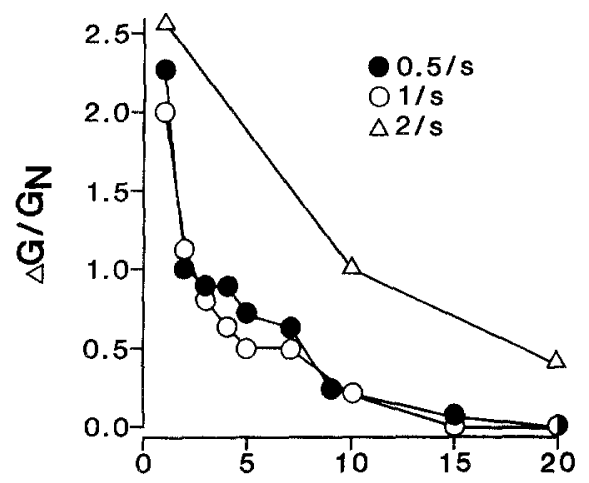

STIMULUS NUMBER
C

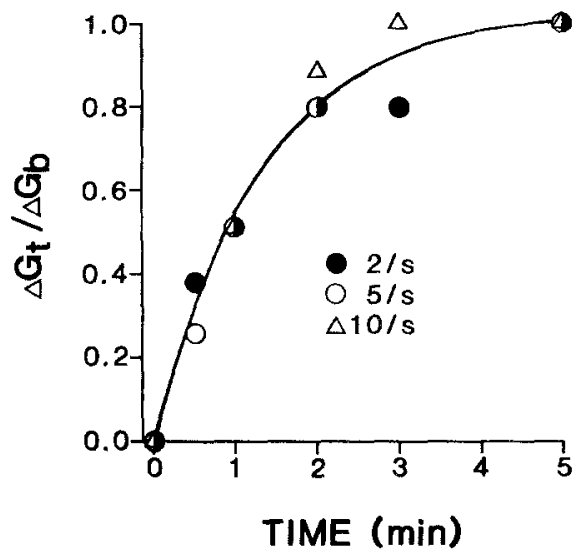

Fig. 6. Stimulation fatigue of 1-IPSPs. (A) Top row: A 30-sec, 2-Hz train of stimuli (2/sec, 2 T) was applied (right; arrows indicate the amplitude of the 1-IPSP evoked by the first stimulus). Pairs of stimuli during this train (number indicated) are shown on the left. Second row: After recovery, another 30-sec train of stimuli (1/sec, $2 T$ ) again reduced the l-IPSP amplitude (left; stimuli 1 and 5 superimposed). After recovery, two 50 -msec current steps that each produced an action potential were applied before (column 2) and poststimulus during each stimulus of another identical stimulus train. Both action potentials are blocked by the first stimulus, however, the second current step produced an action potential by the fifth stimulus (resting $E_{\mathrm{m}}=-68 \mathrm{mV}$ ), indicating that the stimulation fatigue of 1-IPSPs is associated with a fatigue of the conductance increases during l-IPSPs. (B) As an index of the conductance increase during the peak of l-IPSPs, the difference between the membrane conductance determined prestimulus $\left(G_{\mathrm{N}}\right)$ and that measured during the peak of the l-IPSP was calculated for another neuron. The ratio of this $\Delta G$ to $G_{\mathrm{N}}$ is plotted vs the stimulus number for trains of 20 stimuli applied at three different frequencies. Complete fatigue $(\Delta G=0)$ occurs on the 20th and 15 th stimuli for frequencies of 0.5 and $1 \mathrm{~Hz}$, respectively. Increasing the stimulation frequency to $2 \mathrm{~Hz}$ results in a reduction of the degree of fatigue as a function of the stimulus number. (C) Recovery from stimulation fatigue. A $\Delta G$ value was calculated before $\left(\Delta G_{b}\right)$ and at various times after $\left(\Delta G_{t}\right)$ a train of 50 stimuli applied at a frequency of either 2,5 , or $10 \mathrm{~Hz}$. The ratio $\Delta G_{t} / \Delta G_{\mathrm{b}}$ is plotted as a function of the time after the end of the stimulus train for each frequency. Complete fatigue $\left(\Delta G_{t}\right.$ and $\left.\Delta G_{t} / \Delta G_{\mathrm{b}}=0\right)$ occurs by the 50th stimulus $(t=0)$ for each stimulation frequency. Complete recovery $\left(\Delta G_{t} / \Delta G_{\mathrm{b}}=1\right)$ requires $5 \mathrm{~min}$ after $2-\mathrm{Hz}$ stimulation. Increasing the stimulus frequency to 5 or $10 \mathrm{~Hz}$ did not increase the time required for complete recovery. Stimuli were not applied between the times at which $\Delta G_{t} / \Delta G_{\mathrm{b}}$ was determined (all values depicted). Resting $E_{\mathrm{M}},-86 \mathrm{mV}$; same neuron as in $\mathrm{B}$. 
significantly reduced but the amplitude and duration of 1-IPSPs appeared to be unaffected. At application times $>5 \mathrm{~min}$, the concentrations of bicuculline we applied blocked f-IPSPs completely and bursts of action potentials were produced by stimulus intensities that, before the application of bicuculline, were subthreshold for the evocation of 1-IPSPs. Consequently, at stimulus intensities that had evoked 1-IPSPs, 1-IPSPs either were obscured by these bursts or could not be distinguished reliably from the burst after hyperpolarization. That l-IPSPs were still present was suggested, however, by observations that the number of spikes during these bursts of action potentials decreased, rather than increased, as the stimulus intensity was increased over the range of intensities at which 1-IPSPs began to be recruited before the bicuculline application.

Newberry and Nicoll (1984) proposed that the long-duration, potassiumdependent IPSPs they and others have recorded from hippocampal CA1 neurons (slow IPSPs) may be secondary to activation of $\mathrm{GABA}_{\mathrm{B}}$ receptors. This suggestion was based primarily on the similarities between baclofen-induced hyperpolarizations and slow IPSPs. Although baclofen also produces potassium-dependent hyperpolarizations of rat neocortical neurons, baclofen markedly reduced the amplitude of 1-IPSPs in each of 17 neurons tested. Baclofen's depressions of 1-IPSPs were voltage independent at $E_{\mathrm{m}}$ values of between -50 and $-110 \mathrm{mV}$ and we have shown that baclofen reduces the magnitude and duration of the conductance increases during 1-IPSPs [for illustration and further details, see Howe et al. (1987)]. The $\mathrm{EC}_{50}$ for baclofen's action to depress l-IPSPs is approximately $1 \mu M$ and L-baclofen is $>100$ fold more potent than D-baclofen at producing these depressions (Howe et al., 1987; Howe and Zieglgänsberger, 1986).

\section{DISCUSSION}

Stimulation of superficial cortical layers evokes IPSPs that consist of two components in neurons in layers 2 and 3 of the rat frontal neocortex. The conductance increases associated with the second component can exceed $1 \mathrm{sec}$ in duration. We conclude, as have other workers for similar potentials evoked in other CNS neurons, that this second component is not a consequence of preceding depolarizing potentials (Constanti et al., 1980; Satou et al., 1982; Alger, 1984; Newberry and Nicoll, 1984; Thalmann, 1984) but, rather, that it represents a long-duration IPSP which overlaps with, but is distinguishable from, the preceding $\mathrm{GABA}_{\mathrm{A}}$ receptor-mediated f-IPSPs we have described before (Sutor and Zieglgänsberger, 1984; Sutor, 1986).

Our results indicate that increases in postsynaptic potassium conductance make a major contribution to the generation of 1-IPSPs. The changes in the reversal potential of 1-IPSPs that resulted from changes in the extracellular potassium concentration are, however, only about $50 \%$ of those expected for a potential produced solely by the transmembrane movement of potassium ions. In addition, in five neurons, 1-IPSPs were 1-2 $\mathrm{mV}$ depolarizing at resting $E_{\mathrm{m}}$. On the basis of our present results, it is unclear whether this indicates that an ion other than potassium carries part of the currents that underlie l-IPSPs or that the apparent reversal potential of 1-IPSPs is influenced by the preceding chloride-dependent f-IPSPs. 
Although the $E_{\mathrm{IPSP}}$ values of f-IPSPs were shifted to a much lesser extent by changes in the extracellular potassium concentration, the small changes that we observed were statistically significant. It is, however, our opinion that these results do not argue that potassium ions carry a significant portion of the currents that generate f-IPSPs. The $E_{\mathrm{IPSP}}$ determinations were performed with stimulation intensities that were suprathreshold for the generation of action potentials and the peak amplitudes of f-IPSPs coincide approximately with the peak amplitudes of the potassium-dependent spike afterhyperpolarizations. We have noted here that the presence of preceding action potentials had little influence on 1-IPSPs but did influence somewhat the amplitudes of f-IPSPs, and we have noted in other experiments that the $E_{\text {IPSP }}$ values determined for f-IPSPs evoked with subthreshold intensities are 2-3 mV more positive than those determined for f-IPSPs evoked in the same neurons with suprathreshold intensities. It is therefore our opinion that the observed potassium dependence of f-IPSPs is only apparent and results because f-IPSPs overlap in time with the spike afterhyperpolarization and possibly also with the early portion of 1-IPSPs.

The duration of 1-IPSPs decrease as they are evoked at increasingly depolarized $E_{\mathrm{m}}$ values. Long-duration, potassium-dependent IPSPs evoked in other mammalian CNS neurons also become shorter as $E_{\mathrm{m}}$ is depolarized. Satou et al. (1982) concluded that this indicated that the long-duration IPSP conductance was voltage dependent, whereas other authors attributed this to the rectification behavior of the neurons (Constanti et al., 1980; Alger, 1984; Knowles et al., 1984).

We have tried to minimize the influence of the rectification behavior of rat neocortical neurons by determining the depolarizing current just necessary to produce an action potential at different $E_{\mathrm{m}}$ values and then measuring the duration of the poststimulus block of these action potentials as an indicator of the duration of 1-IPSPs. Our results indicate that the durations of the conductance increases during 1-IPSPs decrease with depolarization and increase with hyperpolarization. The voltage dependence of the duration of the conductance increases was quite steep and was clearly demonstrated for each neuron tested. The results are equivocal, however, with regard to whether the 1-IPSP conductance is inwardly rectifying, because the conductance increases measured at poststimulus times of between 200 and $400 \mathrm{msec}$, times when l-IPSPs are fully activated, did not appear to be voltage dependent. In addition, provided that 1-IPSPs evoked at each $E_{\mathrm{m}}$ value were measured at peak amplitude, reversal potential plots were linear at $E_{\mathrm{m}}$ values of between -55 and $-85 \mathrm{mV}$.

Although it is conceivable that the peak IPSP conductance might be voltage independent, but its duration voltage dependent (e.g., if the mean duration, but not the mean amplitude, of the unitary conductance event decreased with depolarization), the interpretation of the results is again complicated by the presence of preceding and overlapping f-IPSPs. Our results indicate that the conductance increases measured during the peak amplitude of f-IPSPs exhibit outward rectification, as do conductance increases produced by iontophoretic applications of GABA or muscimol on rat neocortical neurons. Thus, at early poststimulus times, any possible inward rectification of the 1-IPSP conductance may be counteracted by the outward rectification exhibited by the f-IPSP conductance. Furthermore, with the suprathreshold stimulus intensities we commonly employed, $E_{\mathrm{m}}$ was virtually 
"clamped" $20-30 \mathrm{msec}$ poststimulus at the f-IPSP reversal potential, and thus the onset of 1-IPSPs occurred from roughly the same $E_{\mathrm{m}}$ value regardless of the $E_{\mathrm{m}}$ value at which the stimuli were applied.

Our results do indicate, however, that the decreased duration of 1-IPSPs as $E_{\mathrm{m}}$ is depolarized is due to a decrease in the duration of the conductance increases during 1-IPSPs and is not the result of nonlinearities in the current-voltage relations of rat neocortical neurons. In fact, just the opposite voltage dependence of 1-IPSP durations would be expected, if the changes in duration were simply the result of these nonlinearities. Over the range of $E_{\mathrm{m}}$ values investigated $(-55$ to $-95 \mathrm{mV})$, the current-voltage relations of rat neocortical neurons display inward rectification (Howe et al., 1987; Sutor, 1986), and the increase in resting $R_{\mathrm{N}}$ with depolarization would be expected to increase the amplitude and duration of l-IPSPs, not to decrease them.

The shortening of the time-to-peak amplitude of hyperpolarizing 1-IPSPs was a very obvious and consistent experimental result. This is probably a reflection primarily of the decreased duration of l-IPSPs as $E_{\mathrm{m}}$ is depolarized. The reasons for the shortening of the time-to-peak amplitude of 1-IPSPs as the extracellular potassium concentration is reduced are not readily apparent to us. In addition to increasing the driving force for the generation of hyperpolarizing l-IPSPs, reductions in the extracellular potassium concentration are associated with significant increases in the resting $R_{\mathrm{N}}$ of rat neocortical neurons.

As observed for long-duration, potassium-dependent IPSPs evoked in neurons in other brain regions, 1-IPSPs exhibit stimulation fatigue which is already evident by the second stimulus(Constanti et al., 1980; Connors et al., 1982; Galvan et al., 1982; Alger, 1984). Our results demonstrate that the reduction of 1-IPSP amplitudes with repetitive stimulation is due to a reduction in the conductance increases measured during 1-IPSPs. As for long-duration IPSPs evoked in hippocampal pyramidal neurons (Alger, 1984), recovery from complete stimulation fatigue of 1-IPSPs requires several minutes. Our results indicate that the recovery of 1-IPSP amplitudes is paralleled by the recovery of the conductance increases associated with 1-IPSPs, which additionally supports the conclusion that the stimulation fatigue of the conductance increases associated with 1-IPSPs accounts completely for the fatigue of 1-IPSP amplitudes and durations. Stimulation fatigue of f-IPSPs, however, is due both to a reduction of the

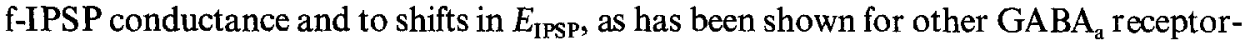
mediated IPSPs (McCarren and Alger, 1985).

Our observations that the maximum extent of stimulation fatigue of 1-IPSPs is attained with the relatively low stimulus frequency of $1 \mathrm{~Hz}$ suggest that the fatigue is not due simply to the failure of the presynaptic fibers to follow the applied stimuli. At 1 $\mathrm{Hz}$ stimulation, changes in resting $E_{\mathrm{m}}$ during the stimulus trains were absent or $\leqslant 1 \mathrm{mV}$, suggesting that this stimulus frequency does not result in significant extracellular accumulation of potassium, which might be expected to decrease presynaptic excitability and lead to less efficient transmitter release. The slow recovery from stimulation fatigue is also inconsistent with either of these possible explanations. It also seems unlikely to us that depletion of presynaptic transmitter stores would be complete after as few as 15 to 20 stimuli, and such a mechanism would not account for the decrease in the extent of stimulation fatigue that was observed as the stimulus 
frequency was increased beyond $1 \mathrm{~Hz}$. On the basis of the present results, however, it is impossible to decide between other possible presynaptic or postsynaptic mechanisms.

Long-duration, potassium-dependent IPSPs evoked in hippocampal neurons have been reported to be resistant to blockade, or even to be enhanced, by bicuculline or picrotoxin (Thalmann and Ayala, 1982; Alger, 1984; Newberry and Nicoll, 1984). In our experiments, applications of bicuculline that markedly reduce f-IPSPs did not appear to reduce 1-IPSPs. As noted above, however, quantitative estimates of the relative sensitivity of f-IPSPs and 1-IPSPs to blockade by bicuculline are complicated by the bursting evoked in rat neocortical neurons at low stimulation intensities during bicuculline applications, as was also noted in the study by Knowles et al. (1984) in hippocampal neurons.

Given the proposed $\mathrm{GABA}_{\mathrm{B}}$-receptor nature of long-duration, potassiumdependent IPSPs in the hippocampus (Newberry and Nicoll, 1984), it is of particular interest that the GABA $\mathrm{B}_{\mathrm{B}}$-agonist baclofen blocks these 1-IPSPs. We have shown that baclofen's depressions of l-IPSPs are voltage independent and that, at the same time baclofen increases resting neuronal input conductance, the conductance increases during 1-IPSPs are reduced (Howe et al., 1987). Thus baclofen's reductions of 1-IPSPs are not due to a postsynaptic shunt of 1-IPSPs secondary to baclofen's action to increase the potassium conductance of rat neocortical neurons. Although one possible explanation for these results is that baclofen reduces presynaptic transmitter release, if baclofen and the transmitter responsible for generation of 1-IPSPs each activate the same postsynaptic potassium conductance, then, as we have discussed previously (Howe et al., 1987), baclofen's depression of 1-IPSPs might also be due in part to a direct postsynaptic competition for this conductance. At present this proposal cannot be tested directly because of the lack of an established antagonist of either baclofeninduced hyperpolarizations or long-duration, potassium-dependent IPSPs.

The neurotransmitter responsible for generation of 1-IPSPs is unknown, but 1-IPSPs are very similar to long-duration IPSPs recorded from neurons in other brain regions, suggesting that these IPSPs represent a relatively common inhibitory mechanism in the mammalian CNS. Recent experiments in our laboratory indicate that 1-IPSPs with properties similar to those described here can be evoked in rat frontal neocortical neurons as early as the ninth postnatal day.

\section{ACKNOWLEDGMENTS}

We wish to thank Ms. V. Grant for her help in typing the manuscript. This work was supported by grants from the BMFT and SFB 200 to W.Z. During revision of the paper, J.R.H. was supported by NRSA Fellowship NS-07370.

\section{REFERENCES}

Alger, B. E. (1984). Characteristics of a slow hyperpolarizing synaptic potential in rat hippocampal pyramidal cells in vitro. $J$. Neurophysiol. 52:892-910.

Connors, B. W., Gutnick, M. J., and Prince, D. A. (1982). Electrophysiological properties of neocortical neurons in vitro. $J$. Neurophysiol. 38:1302-1320. 
Constanti, A., Connor, J. D., Galvan, M., and Nistri, A. (1980). Intracellularly-recorded effects of glutamate and aspartate on neurones in the guinea-pig olfactory cortex slice. Brain Res. 195:403-420.

Fujita, Y. (1979). Evidence for the existence of inhibitory postsynaptic potentials in dendrites and their functional significance in hippocampal pyramidal cells of adult rabbits. Brain Res. 175:59-69.

Galvan, M., Grafe, P., and ten Bruggencate, G. (1982). Convulsant actions of 4-aminopyridine on the guinea-pig olfactory cortex slice. Brain Res. 241:75-86.

Hotson, J. R., and Prince, D. A. (1980). A calcium-activated hyperpolarization follows repetitive firing in hippocampal neurons. J. Neurophysiol. 43:409-419.

Howe, J. R., and Zieglgänsberger, W. (1986). D-Baclofen does not antagonize the actions of L-baclofen on rat neocortical neurons in vitro. Neurosci. Lett. 72: 99-104.

Howe, J. R., Sutor, B., and Zieglgänsberger, W. (1987). Baclofen reduces postsynaptic potentials of rat neocortical neurons by an action other than its hyperpolarizing action. J. Physiol. (Lond.) 384:539-569.

Knowles, W. D., Schneiderman, J. H., Wheal, H. V., Stafstrom, C. E., and Schwartzkroin, P. A. (1984). Hyperpolarizing potentials in guinea-pig hippocampal CA3 neurons. Cell. Mol. Neurobiol. 4:207-230.

McCarren, M., and Alger, B. E. (1985). Use-dependent depression of IPSPs in rat hippocampal pyramidal cells in vitro. $J$. Neurophysiol. 53:557-571.

Newberry, N. R., and Nicoll, R. A. (1984). A bicuculline-resistant inhibitory post-synaptic potential in rat hippocampal pyramidal cells in vitro. J. Physiol. (Lond.) 348:239-254.

Satou, M., Mori, K., Tazawa, Y., and Takagi, S. F. (1982). Two types of postsynaptic inhibition in pyriform cortex of the rabbit: Fast and slow inhibitory postsynaptic potentials. J. Neurophysiol. 48:1142-1156.

Schwartzkroin, P. A., and Stafstrom, C. E. (1980). Effects of EGTA on the calcium-activated afterhyperpolarization in hippocampal CA3 pyramidal cells. Science 210:1125-1126.

Sutor, B. (1986). Nachweis eines GABA-vermittelten, inhibitorischen postsynaptischen Potentials in Neuronen des Neokortex der Ratte, Ph.D. thesis, University of Erlangen-Nürnberg.

Sutor, B., and Zieglgänsberger, W. (1984). A GABA-mediated, chloride-dependent depolarizing IPSP in neocortical neurons of the rat in vitro. Pflïgers Arch. Suppl. 400:R37.

Thalmann, R. H. (1984). Reversal properties of an EGTA-resistant late hyperpolarization that follows synaptic stimulation of hippocampal neurons. Neurosci. Lett. 46:103-108.

Thalmann, R. H., and Ayala, G. F. (1982). A late increase in potassium conductance follows synaptic stimulation of granule neurons of the dentate gyrus. Neurosci. Lett. 29:243-248. 